\title{
Antiperiodic Boundary Value Problem for Second-Order Impulsive Differential Equations on Time Scales
}

\author{
Yepeng Xing, Qiong Wang, and De Chen \\ Department of Applied Mathematics, Shanghai Normal University, Shanghai 200234, China \\ Correspondence should be addressed to Yepeng Xing, ypxing-jason@hotmail.com
}

Received 3 April 2009; Accepted 20 July 2009

Recommended by Alberto Cabada

We prove the existence results for second-order impulsive differential equations on time scales with antiperiodic boundary value conditions in the presence of classical fixed point theorems.

Copyright (c) 2009 Yepeng Xing et al. This is an open access article distributed under the Creative Commons Attribution License, which permits unrestricted use, distribution, and reproduction in any medium, provided the original work is properly cited.

\section{Introduction and Preliminaries}

Many evolution processes are characterized by the fact that at certain moments of time they experience a change of state abruptly. Consequently, it is natural to assume that these perturbations act instantaneously, that is, in the form of impulses. It is known that many biological phenomena involving threshold, bursting rhythm models in medicine and biology, optimal control models in economics, pharmacokinetics, and frequency modulated systems do exhibit impulse effects. The branch of modern applied analysis known as "impulsive" differential equations provides a natural framework to mathematically describe the aforementioned jumping processes. The reader is referred to monographs [1-4] and references therein for some nice examples and applications to the above areas.

The study of dynamic equations on time scales goes back to Stefan Hilger [5]. Now it is still a new area of fairly theoretical exploration in mathematics. In the recent years, there has been much progress on the qualitative properties of dynamic systems on time scales, see $[6,7]$. However, there is not so much work on antiperiodic boundary value problems for differential equations on time scales except that in [8] by Cabada In [9-12], the authors studied antiperiodic boundary value problem for ordinary differential equations without impulses, while in [13-18] the authors considered antiperiodic boundary value problem for impulsive differential equations. In this paper, we study two types of second-order impulsive 
differential equations with antiperiodic boundary value conditions on time scales. Firstly, we use Schauder's fixed point theorem to study the existence results of the following boundary value problem:

$$
\begin{gathered}
-x^{\Delta \Delta}(t)=f\left(t, x(t), x^{\Delta}(t)\right), \quad t \in[0, T] \backslash \Omega:=\left\{t_{1}, t_{2}, \ldots, t_{m}\right\}, \\
x\left(t_{k}^{+}\right)=x\left(t_{k}\right)+I_{k}\left(x\left(t_{k}\right)\right), \quad x^{\Delta}\left(t_{k}^{+}\right)=x^{\Delta}\left(t_{k}\right)+J_{k}\left(x\left(t_{k}\right), x^{\Delta}\left(t_{k}\right)\right), \quad k=1,2, \ldots, m, \\
x(0)=-x(\sigma(T)), \quad x^{\Delta}(0)=-x^{\Delta}(\sigma(T)) .
\end{gathered}
$$

Secondly, we employ Schaefer's fixed point to investigate the following antiperiodic boundary value problem on time scales:

$$
\begin{gathered}
x^{\Delta \Delta}(t)=f\left(t, x^{\sigma}(t), x^{\Delta}(t)\right), \quad t \in[0, T] \backslash\left\{t_{1}, t_{2}, \ldots, t_{m}\right\}, \\
x\left(t_{k}^{+}\right)=x\left(t_{k}\right)+I_{k}\left(x\left(t_{k}\right)\right), \quad x^{\Delta}\left(t_{k}^{+}\right)=x^{\Delta}\left(t_{k}\right)+J_{k}\left(x\left(t_{k}\right), x^{\Delta}\left(t_{k}\right)\right), \quad k=1,2, \ldots, m, \\
x(0)=-x(\sigma(T)), \quad x^{\Delta}(0)=-x^{\Delta}(\sigma(T)) .
\end{gathered}
$$

We assume throughout this paper that $f:[0, T] \times \mathbb{R}^{n} \times \mathbb{R}^{n} \rightarrow \mathbb{R}^{n}$ is continuous and $I_{k}: \mathbb{R}^{n} \rightarrow$ $\mathbb{R}^{n}, J_{k}: \mathbb{R}^{n} \times \mathbb{R}^{n} \rightarrow \mathbb{R}^{n}$ are also continuous $(k=1,2, \ldots, m)$.

We should mention that (1.1), (1.2) are more general than equations considered in $[13,18]$ since $f, J$ both contain $x$ and its derivative. So our result is still new even when $(1.1),(1.2)$ reduce to equations studied in $[13,18]$. The paper is organized as follows. In Section 2 we present the expression of Green's functions of related linear operator in the space of piecewise continuous functions. Section 3 contains the main results of the paper and is devoted to the existence of solutions to (1.1) and (1.2). useful.

To understand the concept of time scales and the above notation, some definitions are

A time scale is an arbitrary nonempty closed subset of the real numbers. Throughout this paper, we will denote a time scale by the symbol $\mathbb{T}$. And the forward and backward jump operators $\sigma, \rho: \mathbb{T} \rightarrow \mathbb{T}$ are defined by

$$
\sigma(t)=\inf \{s \in \mathbb{T}: s>t\}, \quad \rho(t)=\sup \{s \in \mathbb{T}: s<t\}
$$

respectively. The point $t \in \mathbb{T}$ is called left dense, left scattered, right dense, or right-scattered if $\rho(t)=t, \rho(t)<t, \sigma(t)=t$ or $\sigma(t)>t$, respectively. Points that are right dense and left dense at the same time are called dense. We denote $\sigma(\sigma(t))$ by $\sigma^{2}(t)$.

If $\mathbb{T}$ has a left scattered maximum $m$, define $\mathbb{T}^{k}:=\mathbb{T}-\{m\}$; otherwise, set $\mathbb{T}^{k}=\mathbb{T}$. The symbols $[a, b],[a, b)$, and so on, denote time scales intervals, for example,

$$
[a, b]=\{t \in \mathbb{T}: a \leq t \leq b\}
$$

where $a, b \in \mathbb{T}$ with $a<\rho(b)$. 
Definition 1.1. A vector function $f: \mathbb{T} \rightarrow \mathbb{R}^{n}$ is rd-continuous provided that it is continuous at each right dense point in $\mathbb{T}$ and has a left-sided limit at each left dense point in $\mathbb{T}$. The set of rd-continuous functions $f: \mathbb{T} \rightarrow \mathbb{R}^{n}$ will be denoted in this paper by $C_{\mathrm{rd}}(\mathbb{T})=C_{\mathrm{rd}}\left(\mathbb{T}, \mathbb{R}^{n}\right)$.

Definition 1.2. Assume $f: \mathbb{T} \rightarrow \mathbb{R}$ is a function and let $t \in \mathbb{T}^{k}$. Then we define $f^{\Delta}(t)$ to be the number (provided it exists) with the property that given any $\varepsilon>0$ there exists a neighborhood $U$ of $t$ (i.e., $U=(t-\delta, t+\delta) \cap \mathbb{T}$ for some $\delta>0$ ) such that

$$
\left|f(\sigma(t))-f(s)-f^{\Delta}(t)[\sigma(t)-s]\right| \leq \varepsilon|\sigma(t)-s|, \quad \forall s \in U
$$

We call $f^{\Delta}(t)$ the delta (or Higher) derivative of $f$ at $t$.

Definition 1.3. A function $F: \mathbb{T} \rightarrow \mathbb{R}$ is called an antiderivative of $f: \mathbb{T} \rightarrow \mathbb{R}$ provided

$$
F^{\Delta}(t)=f(t) \quad \text { holds } \forall t \in \mathbb{T} \text {. }
$$

Theorem 1.4 (existence of antiderivatives). Every $r$-continuous function has an antiderivative. In particular if $t_{0} \in \mathbb{T}$, then $F$ defined by

$$
F(t):=\int_{t_{0}}^{t} f(t) \Delta t \quad \text { for } t \in \mathbb{T}
$$

is an antiderivative of $f$.

We assume that for $f\left(t_{k^{+}}^{+} x, y\right):=\lim _{t \rightarrow t_{k}^{+}} f(t, x, y)$ and $f\left(t_{k^{-}}, x, y\right):=\lim _{t \rightarrow t_{k}^{-}} f(t, x, y)$ both exist with $f\left(t_{k}^{-}, x, y\right)=f\left(t_{k}, x, y\right), k=1,2, \ldots, m$. In order to define a solution of (1.1) and (1.2), we introduce and denote the Banach space $P C\left([0, T], \mathbb{R}^{n}\right)$ by

$$
\begin{aligned}
P C\left([0, T] ; \mathbb{R}^{n}\right):=\left\{u:[0, T] \longrightarrow \mathbb{R}^{n}, u \in C\left([0, T] \backslash \Omega, \mathbb{R}^{n}\right),\right. \\
\left.u \text { is left continuous at } t=t_{k}, \text { the right-hand limit } u\left(t_{k}^{+}\right) \text {exists }\right\}
\end{aligned}
$$

with the norm $\|u\|_{P C}:=\sup _{t \in[0, T]}\|u(t)\|$ where $\|\cdot\|$ is the usual Euclidean norm and $\langle\cdot, \cdot\rangle$ will be the Euclidean inner product.

In a similar fashion to the above, define and denote the Banach space $P C^{1}\left([0, T], \mathbb{R}^{n}\right)$ by

$$
\begin{aligned}
& P C^{1}\left([0, T] ; \mathbb{R}^{n}\right):=\left\{u \in P C\left([0, T] ; \mathbb{R}^{n}\right): u^{\Delta}(t) \in C\left([0, T] \backslash \Omega, \mathbb{R}^{n}\right),\right. \\
&\text { the limits } \left.u^{\Delta}\left(t_{k}^{-}\right), u^{\Delta}\left(t_{k}^{+}\right) \text {exist with } u^{\Delta}\left(t_{k}^{-}\right)=u^{\Delta}\left(t_{k}\right)\right\}
\end{aligned}
$$

with the norm $\|u\|_{P C^{1}}:=\sup _{t \in[0, T]}\left\{\|u(t)\|_{P C},\left\|u^{\Delta}(t)\right\|_{P C}\right\}$.

The following fixed point theorem is our main tool to prove the existence of at least one solution to (1.2). 
Theorem 1.5 (Schaefer's fixed point theorem [19]). Let $X$ be a Banach space and let $A: X \rightarrow X$ be a completely continuous operator. Then either:

(i) the operator equation $x=\lambda A x$ has a solution for $\lambda=1$. or

(ii) the set $S:=\{x \in X, x=\lambda A x, \lambda \in] 0,1[\}$ is unbounded.

\section{Expression of Green's Function}

In this part, we present the expression of Green's functions for second-order impulsive equations with antiperiodic conditions.

Lemma 2.1. For any $h(t) \in P C\left([0, T], \mathbb{R}^{n}\right), x(t)$ solves

$$
\begin{gathered}
-x^{\Delta \Delta}=h(t), \quad t \in[0, T] \backslash \Omega, \\
x\left(t_{k}^{+}\right)=x\left(t_{k}\right)+I_{k}\left(x\left(t_{k}\right)\right), \quad x^{\Delta}\left(t_{k}^{+}\right)=x^{\Delta}\left(t_{k}\right)+J_{k}\left(x\left(t_{k}\right), x^{\Delta}\left(t_{k}\right)\right), \quad k=1,2, \ldots, m, \\
x(0)=-x(\sigma(T)), \quad x^{\Delta}(0)=-x^{\Delta}(\sigma(T)),
\end{gathered}
$$

if and only if $x(t)$ is the solution of integral equation

$$
\begin{aligned}
x(t)= & \int_{0}^{\sigma(T)} G(t, \sigma(s)) h(s) \Delta s+\sum_{k=1}^{m} H\left(t, t_{k}\right) I_{k}\left(x\left(t_{k}\right)\right) \\
& -\sum_{k=1}^{m} G\left(t, t_{k}\right) J_{k}\left(x\left(t_{k}\right), x^{\Delta}\left(t_{k}\right)\right), \quad \forall t \in\left[0, \sigma^{2}(T)\right],
\end{aligned}
$$

where

$$
\begin{gathered}
G(t, s)=\left\{\begin{array}{l}
\frac{1}{2}\left[\frac{1}{2} \sigma(T)-t+s\right] \quad 0 \leq s \leq t \leq \sigma^{2}(T), \\
\frac{1}{2}\left[\frac{1}{2} \sigma(T)+t-s\right] \quad 0 \leq t<s \leq \sigma(T),
\end{array}\right. \\
H(t, s)= \begin{cases}\frac{1}{2}, & 0 \leq s \leq t \leq \sigma^{2}(T), \\
-\frac{1}{2}, & 0 \leq t<s \leq \sigma(T) .\end{cases}
\end{gathered}
$$

Proof. Assume $x(t)$ is a solution of (2.1). Then by integrating $-x^{\Delta \Delta}=h(t), t \neq t_{k},(k=$ $1,2, \ldots, m)$ step by step from 0 to $t$ we have

$$
x^{\Delta}(t)=x^{\Delta}(0)-\int_{0}^{t} h(s) \Delta s+\sum_{0<t_{k}<t}\left[x^{\Delta}\left(t_{k}^{+}\right)-x^{\Delta}\left(t_{k}\right)\right], \quad \forall t \in[0, \sigma(T)] .
$$


Similarly, we Integrate (2.3) from 0 to $t$ step by step to get

$$
x(t)=x(0)+\int_{0}^{t} x^{\Delta}(s) \Delta s+\sum_{0<t_{k}<t}\left[x\left(t_{k}^{+}\right)-x\left(t_{k}\right)\right], \quad \forall t \in\left[0, \sigma^{2}(T)\right] .
$$

Substituting (2.3) into (2.4), we obtain

$$
\begin{aligned}
x(t)= & x(0)+x^{\Delta}(0) t-\int_{0}^{t}(t-\sigma(s)) h(s) \Delta s \\
& +\sum_{0<t_{k}<t}\left[x\left(t_{k}^{+}\right)-x\left(t_{k}\right)\right]+\sum_{0<t_{k}<t}\left[x^{\Delta}\left(t_{k}^{+}\right)-x^{\Delta}\left(t_{k}\right)\right]\left(t-t_{k}\right), \quad \forall t \in\left[0, \sigma^{2}(T)\right] .
\end{aligned}
$$

By $x^{\Delta}(0)=-x^{\Delta}(\sigma(T))$ and $x^{\Delta}\left(t_{k}^{+}\right)=x^{\Delta}\left(t_{k}\right)+J_{k}\left(x\left(t_{k}\right), x^{\Delta}\left(t_{k}\right)\right)$, we have from (2.3) that

$$
x^{\Delta}(0)=\frac{1}{2} \int_{0}^{\sigma(T)} h(s) \Delta s-\frac{1}{2} \sum_{k=1}^{m} J_{k}\left(x\left(t_{k}\right), x^{\Delta}\left(t_{k}\right)\right) .
$$

Note that $x(0)=-x(\sigma(T)), x\left(t_{k}^{+}\right)=x\left(t_{k}\right)+I_{k}\left(x\left(t_{k}\right)\right)$ and (2.6) . It then follows from (2.4) that

$$
\begin{aligned}
x(0)= & -\frac{\sigma(T)}{4}\left[\int_{0}^{\sigma(T)} h(s) \Delta s-\sum_{k=1}^{m} J_{k}\left(x\left(t_{k}\right), x^{\Delta}\left(t_{k}\right)\right)\right] \\
& +\frac{1}{2} \int_{0}^{\sigma(T)}(\sigma(T)-s) h(s) \Delta s-\frac{1}{2} \sum_{k=1}^{m} J_{k}\left(x\left(t_{k}\right), x^{\Delta}\left(t_{k}\right)\right)\left[\sigma(T)-t_{k}\right]-\frac{1}{2} \sum_{k=1}^{m} I_{k}\left(x\left(t_{k}\right)\right) .
\end{aligned}
$$

Then we substitute (2.6), (2.7) into (2.5) to obtain

$$
\begin{aligned}
x(t)= & \int_{0}^{\sigma(T)}\left[\frac{1}{4} \sigma(T)-\frac{1}{2} \sigma(s)+\frac{1}{2} t\right] h(s) \Delta s-\int_{0}^{t}(t-\sigma(s)) h(s) \Delta s \\
& +\sum_{k=1}^{m} J_{k}\left(x\left(t_{k}\right), x^{\Delta}\left(t_{k}\right)\right)\left[-\frac{1}{4} \sigma(T)+\frac{1}{2} t_{k}-\frac{1}{2} t\right] \\
& +\sum_{0<t_{k}<t}\left(t-t_{k}\right) J_{k}\left(x\left(t_{k}\right), x^{\Delta}\left(t_{k}\right)\right)-\frac{1}{2} \sum_{k=1}^{m} I_{k}\left(x\left(t_{k}\right)\right)+\sum_{0<t_{k}<t} I_{k}\left(x\left(t_{k}\right)\right) .
\end{aligned}
$$

If we choose $G(t, s), H(t, s)$ as $(G) H$, then (2.2) is true.

Now assume $x(t)$ is a solution of (2.2). Then for $t \neq t_{k}, k=1,2, \ldots, m$. we have by [6, Theorem 1.117, page 46] that

$$
x^{\Delta}(t)=\int_{0}^{T} G^{\Delta}(t, \sigma(s)) h(s) \Delta s+\sum_{k=1}^{m} H^{\Delta}\left(t, t_{k}\right) I_{k}\left(x\left(t_{k}\right)\right)-\sum_{k=1}^{m} G^{\Delta}\left(t, t_{k}\right) J_{i}\left(x\left(t_{k}\right), x^{\Delta}\left(t_{k}\right)\right),
$$


where $G^{\Delta}, f^{\Delta}$ denotes the derivatives of $G, H$ with respect to the first variable. That is

$$
x^{\Delta}(t)=-\frac{1}{2} \int_{0}^{t} h(s) \Delta s+\frac{1}{2} \int_{t}^{\sigma(T)} h(s) \Delta s+\sum_{k=1}^{m} J_{k}\left(x\left(t_{k}\right), x^{\Delta}\left(t_{k}\right)\right) .
$$

Compute straightforwardly to get

$$
x^{\Delta \Delta}(t)=-h(t)
$$

Since

$$
\sum_{k=1}^{m} H\left(t, t_{k}\right) I_{k}\left(x\left(t_{k}\right)\right)=-\frac{1}{2} \sum_{k=1}^{m} I_{k}\left(x\left(t_{k}\right)\right)+\sum_{0<t_{k}<t} I_{k}\left(x\left(t_{k}\right)\right),
$$

we have

$$
\begin{aligned}
& \sum_{k=1}^{m} H\left(t_{k}^{+}, t_{k}\right) I_{k}\left(x\left(t_{k}\right)\right)-\sum_{k=1}^{m} H\left(t_{k}, t_{k}\right) I_{k}\left(x\left(t_{k}\right)\right) \\
& \quad=\frac{1}{2}\left[\sum_{i=0}^{k} I_{i}\left(x\left(t_{i}\right)\right)-\sum_{i=k+1}^{m} I_{i}\left(x\left(t_{i}\right)\right)\right]-\frac{1}{2}\left[\sum_{i=0}^{k-1} I_{i}\left(x\left(t_{i}\right)\right)-\sum_{i=k}^{m} I_{i}\left(x\left(t_{i}\right)\right)\right]=I_{k}\left(x\left(t_{k}\right)\right) .
\end{aligned}
$$

Similarly,

$$
\begin{gathered}
\sum_{k=1}^{m} G\left(t_{k}^{+}, t_{k}\right) J_{k}\left(x\left(t_{k}\right), x^{\Delta}\left(t_{k}\right)\right)-\sum_{k=1}^{m} G\left(t_{k}, t_{k}\right) J_{k}\left(x\left(t_{k}\right), x^{\Delta}\left(t_{k}\right)\right)=0, \\
\sum_{k=1}^{m} G^{\Delta}\left(t_{k}^{+}, t_{k}\right) J_{k}\left(x\left(t_{k}\right), x^{\Delta}\left(t_{k}\right)\right)-\sum_{k=1}^{m} G^{\Delta}\left(t_{k}, t_{k}\right) J_{k}\left(x\left(t_{k}\right), x^{\Delta}\left(t_{k}\right)\right)=-J_{k}\left(x\left(t_{k}\right), x^{\Delta}\left(t_{k}\right)\right) .
\end{gathered}
$$

Thus, the proof is completed.

By Lemma 2.1, we have for every $t \in[0, \sigma(T)]$

$$
\max _{(t, s) \in[0, \sigma(T)] \times[0, T]}|G(t, \sigma(s))| \leq \frac{3}{4} \sigma(T) .
$$

If $t=\sigma^{2}(T)>\sigma(T)$, then

$$
\left|G\left(\sigma^{2}(T), \sigma(s)\right)\right| \leq \frac{1}{4}\left[\sigma(T)+2 \sigma^{2}(T)\right] .
$$


Therefore we have the upper bounds

$$
\begin{gathered}
\max _{\left.(t, s) \in\left[0, \sigma^{2}(T)\right] \times\left[0, \sigma^{(} T\right)\right]}|G(t, \sigma(s))| \leq \frac{1}{4}\left[\sigma(T)+2 \sigma^{2}(T)\right]:=G_{0}, \\
\max _{\left.(t, s) \in\left[0, \sigma^{2}(T)\right] \times\left[0, \sigma^{(} T\right)\right]}|H(t, s)|=\frac{1}{2}, \\
\max _{\left.(t, s) \in\left[0, \sigma^{2}(T)\right] \times\left[0, \sigma^{(} T\right)\right]}\left|G^{\Delta}(t, s)\right|=\frac{1}{2} .
\end{gathered}
$$

Recall that a mapping between Banach spaces is compact if it is continuous and carries bounded sets into relatively compact sets.

Lemma 2.2. Suppose that $f:[0, T] \times \mathbb{R}^{n} \times \mathbb{R}^{n} \rightarrow \mathbb{R}^{n}, I_{k}: \mathbb{R}^{n} \rightarrow \mathbb{R}^{n}$, and $J_{k}: \mathbb{R}^{n} \times \mathbb{R}^{n} \rightarrow \mathbb{R}^{n}$ are continuous. Define an operator $A: P C^{1}\left(\left[0, \sigma^{2}(T)\right], \mathbb{R}^{n}\right) \rightarrow P C^{1}\left(\left[0, \sigma^{2}(T)\right], \mathbb{R}^{n}\right)$ as

$$
\begin{aligned}
A x(t):= & \int_{0}^{\sigma(T)} G(t, \sigma(s)) f\left(s, x(s), x^{\Delta}(s)\right) \Delta s+\sum_{k=1}^{m} H\left(t, t_{k}\right) I_{k}\left(x\left(t_{k}\right)\right) \\
& -\sum_{k=1}^{m} G\left(t, t_{k}\right) J_{k}\left(x\left(t_{k}\right), x^{\Delta}\left(t_{k}\right)\right)
\end{aligned}
$$

where $G(t, s)$ and $H(t, s)$ are as given in Lemma 2.1. Then $A$ is a compact map.

Proof. From (2.10) we know

$$
(A x)^{\Delta}(t)=-\frac{1}{2} \int_{0}^{t} f\left(s, x(s), x^{\Delta}(s)\right) \Delta s+\frac{1}{2} \int_{t}^{\sigma(T)} f\left(s, x(s), x^{\Delta}(s)\right) \Delta s+\sum_{k=1}^{m} J_{k}\left(x\left(t_{k}\right), x^{\Delta}\left(t_{k}\right)\right) .
$$

Then the continuality of $f, I_{k}, J_{k}$ implies $A$ is a continuous map from $P C\left[J, \mathbb{R}^{n}\right]$ to $P C\left[J, \mathbb{R}^{n}\right]$. On the other hand, for any bounded subset $S \subset P C\left[J, \mathbb{R}^{n}\right] .(2.19)$ implies $\left\{(A x)^{\Delta}(t) \mid x(t) \in S\right\}$ is also a bounded subset of $P C\left[J, \mathbb{R}^{n}\right]$. Deducing in a similar way as proving [20, Lemma 2.4], we have $A$ is a compact map.

Lemma 2.3. $x \in P C^{1}\left[J, \mathbb{R}^{n}\right] \cap P C^{2}\left[J, \mathbb{R}^{n}\right]$ is a solution of (1.1) if and only if $x(t) \in P C^{1}\left[J, \mathbb{R}^{n}\right]$ is a fixed point of $A$.

Proof. It can be easily obtain from Lemma 2.1, and we omit the proof here.

\section{Main Results}

In this section, we prove the existence results for (1.1), (1.2) in presence of Schauder's fixed point theorem and Schaefer's fixed point theorem, respectively. 
We first set

$$
\begin{gathered}
\alpha=\lim _{\|x\|+\|y\| \rightarrow \infty}\left(\max _{t \in[0, T]} \frac{\|f(t, x, y)\|}{\|x\|+\|y\|}\right), \\
\beta_{k}=\lim _{\|x\| \rightarrow \infty}\left(\frac{\left\|I_{k}(x)\right\|}{\|x\|}\right) \quad(k=1,2, \ldots, m), \\
\gamma_{k}=\frac{\lim _{\|x\|+\|y\| \rightarrow \infty}\left(\frac{\left\|J_{k}(x, y)\right\|}{\|x\|+\|y\|}\right) \quad(k=1,2, \ldots, m) .}{}
\end{gathered}
$$

Theorem 3.1. Suppose that $f:[0, T] \times \mathbb{R}^{n} \times \mathbb{R}^{n} \rightarrow \mathbb{R}^{n}, I_{k}: \mathbb{R}^{n} \rightarrow \mathbb{R}^{n}$ and $J_{k}: \mathbb{R}^{n} \times \mathbb{R}^{n} \rightarrow \mathbb{R}^{n}$ are continuous $(k=1,2, \ldots, m)$. If

$$
\eta=\max \left\{\eta_{1}, \eta_{2}\right\}<1
$$

where

$$
\begin{gathered}
\eta_{1}=\frac{1}{2}\left[\alpha \sigma(T)+\sum_{k=1}^{m} \gamma_{k}\right]\left[\sigma(T)+2 \sigma^{2}(T)\right]+\frac{1}{2} \sum_{k=1}^{m} \beta_{k}, \\
\eta_{2}=\alpha \sigma(T)+\sum_{k=1}^{m} \gamma_{k} .
\end{gathered}
$$

then (1.1) has at least one solution in $P C^{1}\left[J, \mathbb{R}^{n}\right] \cap P C^{2}\left[J, \mathbb{R}^{n}\right]$.

Proof. By Lemma 2.2 it is sufficient to show that $A$ has at least one solution in $P C^{1}\left[J, \mathbb{R}^{n}\right]$. First, we can choose by (3.2) $\alpha^{\prime}>\alpha, \beta_{k}^{\prime}>\beta_{k}, \gamma_{k}^{\prime}>\gamma_{k},(k=1,2, \ldots, m)$ such that

$$
\begin{gathered}
\eta_{1}^{\prime}=\frac{1}{2}\left[\alpha^{\prime} \sigma(T)+\sum_{k=1}^{m} r_{k}^{\prime}\right]\left[\sigma(T)+2 \sigma^{2}(T)\right]+\frac{1}{2} \sum_{k=1}^{m} \beta_{k}^{\prime}<1, \\
\eta_{2}^{\prime}=\alpha^{\prime} \sigma(T)+\sum_{k=1}^{m} r_{k}^{\prime}<1 .
\end{gathered}
$$

By (3.1) we can choose a positive number $N$ such that

$$
\|f(t, x, y)\|<\alpha^{\prime}(\|x\|+\|y\|), \quad \forall t \in[0, \sigma(T)],\|x\|+\|y\| \geq N
$$

Then

$$
\|f(t, x, y)\| \leq \alpha^{\prime}(\|x\|+\|y\|)+M, \quad \forall t \in[0, \sigma(T)], x, y \in \mathbb{R}^{N},
$$


where

$$
M=\max _{t \in[0, \sigma(T)],\|x\|+\|y\| \leq N}\|f(t, x, y)\|<\infty
$$

Similarly, there exist positive constants $M_{k}, \bar{M}_{k},(k=1,2, \ldots, m)$ such that

$$
\begin{gathered}
\left\|I_{k}(x)\right\| \beta_{k}^{\prime}\|x\|+M_{k}, \quad \forall x \in \mathbb{R}^{n}, \\
\left\|J_{k}(x, y)\right\| \leq \gamma_{k}^{\prime}(\|x\|+\|y\|)+\bar{M}_{k}, \quad \forall x, y \in \mathbb{R}^{n} .
\end{gathered}
$$

It then follows by $(2.17),(2.18),(3.6)-(3.9)$ that

$$
\begin{aligned}
\|(A x)(t)\| \leq & \frac{1}{4} \sigma(T)\left[\sigma(T)+2 \sigma^{2}(T)\right]\left[\alpha^{\prime}\left(\|x(t)\|+\left\|x^{\Delta}(t)\right\|\right)+M\right] \\
& +\frac{1}{2}\left(\sum_{k=1}^{m} \beta_{k}^{\prime}\|x\|+\sum_{k=1}^{m} M_{k}\right) \\
& +\frac{1}{4} \sigma(T)\left[\sigma(T)+2 \sigma^{2}(T)\right]\left[\sum_{k=1}^{m} \gamma_{k}^{\prime}\left(\|x(t)\|+\left\|x^{\Delta}(t)\right\|\right)+\sum_{k=1}^{m} \bar{M}_{k}\right] \\
\leq & \eta_{1}^{\prime}\|x\|_{P C^{1}}+M^{(1)}, \quad \forall t \in\left[0, \sigma^{2}(T)\right],
\end{aligned}
$$

where

$$
M^{(1)}=\frac{1}{4} \sigma(T)\left[\sigma(T)+2 \sigma^{2}(T)\right]\left(M+\sum_{k=1}^{m} \bar{M}_{k}\right)+\frac{1}{2}\left(\sum_{k=1}^{m} M_{k}\right)
$$

is a constant.

Similarly, we can prove that

$$
\left\|(A x)^{\Delta}(t)\right\| \leq \eta_{2}^{\prime}\|x\|_{P C^{1}}+M^{(2)}, \quad \forall t \in\left[0, \sigma^{2}(T)\right],
$$

where $M^{(2)}=(1 / 2)\left(M+\sum_{k=1}^{m} \bar{M}_{k}\right)$ is a constant.

Consequently, by (3.10) and (3.12) we have

$$
\|A x\|_{P C^{1}} \leq \eta^{\prime}\|x\|_{P C^{1}}+M^{\prime}, \quad \forall x \in P C^{1}\left[\left[0, \sigma^{2}(T)\right], \mathbb{R}^{n}\right]
$$

where

$$
\eta^{\prime}=\max \left\{\eta_{1}^{\prime}, \eta_{2}^{\prime}\right\}<1, \quad M^{\prime}=\max \left\{M^{(1)}, M^{(2)}\right\}
$$


Thus we can choose $r>0$ such that

$$
A\left(B_{r}\right) \subset B_{r}
$$

where $B_{r}=\left\{x \in P C^{1}\left[\left[0, \sigma^{2}(T)\right], \mathbb{R}^{n}\right] \mid\|x\|_{P C^{1}} \leq r\right\}$. On the other hand, from Lemma $2.3 A$ is a complete continuous operator. Hence by Schauder's fixed point theorem $A$ has fixed point in $B_{r}$. This completes the proof.

Remark 3.2. Assumption (3.2) is true if there hold

$$
\begin{gathered}
\frac{\|f(t, x, y)\|}{\|x\|+\|y\|} \rightrightarrows 0, \quad \frac{\left\|J_{k}(x, y)\right\|}{\|x\|+\|y\|} \longrightarrow 0, \quad \text { as }\|p\|+\|q\| \longrightarrow \infty \\
\frac{\left\|I_{k}(x)\right\|}{\|x\|} \longrightarrow 0, \quad \text { as }\|x\| \longrightarrow \infty(k=1,2, \ldots, m) .
\end{gathered}
$$

We now go on to study the existence results for (1.2). We should mention that the idea used in the following theorem is initiated by Tisdell [21, 22].

Theorem 3.3. Suppose that $f:[0, T] \times \mathbb{R}^{n} \times \mathbb{R}^{n} \rightarrow \mathbb{R}^{n}, I_{k}: \mathbb{R}^{n} \rightarrow \mathbb{R}^{n}$ and $J_{k}: \mathbb{R}^{n} \times \mathbb{R}^{n} \rightarrow \mathbb{R}^{n}$ are continuous $(k=1,2, \ldots, m)$. If there exist nonnegative constants $\gamma, \delta_{k}, \zeta_{k}, L_{k}, N_{k}$ and $M$ such that

$$
\begin{gathered}
\|f(t, x, y)\| \leq r\left[\langle x, f(t, x, y)\rangle+\|y\|^{2}\right]+M, \quad \forall(t, x, y) \in\left([0, \sigma(T)] \backslash \Omega \times \mathbb{R}^{n} \times \mathbb{R}^{n}\right), \\
\left\|I_{k}(x)\right\| \leq \delta_{k}\|x\|+L_{k}, \quad\left\|J_{k}(x, y)\right\| \leq \zeta_{k}(\|x\|+\|y\|)+N_{k}, \quad \forall x, y \in \mathbb{R}^{n}, \\
\max \left\{\frac{1}{2} \sum_{k=1}^{m} \delta_{i}+2 G_{0} \sum_{i=1}^{m} \zeta_{i}, \sum_{i=1}^{m} \zeta_{i}\right\}<1,
\end{gathered}
$$

where $\langle\cdot\rangle$ is the Euclidean inner product. Then (1.2) has at least one solution.

Proof. Consider the mapping

$$
\begin{aligned}
A & : P C^{1}\left(\left[0, \sigma^{2}(T)\right], \mathbb{R}^{n}\right) \longrightarrow P C^{1}\left(\left[0, \sigma^{2}(T)\right], \mathbb{R}^{n}\right) \\
A x(t)= & \int_{0}^{\sigma(T)} G(t, \sigma(s)) f\left(s, x^{\sigma}(s), x^{\Delta}(s)\right) \Delta s+\sum_{k=1}^{m} H\left(t, t_{k}\right) I_{k}\left(x\left(t_{k}\right)\right) \\
& +\sum_{k=1}^{m} G\left(t, t_{k}\right) J_{k}\left(x\left(t_{k}\right), x^{\Delta}\left(t_{k}\right)\right),
\end{aligned}
$$

where

$$
G(t, s)=\left\{\begin{aligned}
-\frac{1}{2}\left[\frac{1}{2} \sigma(T)-t+s\right] & 0 \leq s \leq t \leq \sigma^{2}(T) \\
-\frac{1}{2}\left[\frac{1}{2} \sigma(T)+t-s\right] & 0 \leq t<s \leq \sigma(T)
\end{aligned}\right.
$$




$$
H(t, s)=G^{\Delta}(t, s) \begin{cases}\frac{1}{2}, & 0 \leq s \leq t \leq \sigma^{2}(T) \\ -\frac{1}{2}, & 0 \leq t<s \leq \sigma(T) .\end{cases}
$$

In a similar way as we prove Lemmas 2.1,2.2 and 2.3 we obtain

(i) $x \in P C^{1}\left[J, \mathbb{R}^{n}\right] \cap P C^{2}\left[J, \mathbb{R}^{n}\right]$ is a solution of (1.2) if and only if $x(t) \in P C^{1}\left[J, \mathbb{R}^{n}\right]$ is a fixed point of $A$;

(ii) $A$ is a compact operator.

Consider the equation

$$
x=A x .
$$

To show $A$ has at least one fixed point, we apply Schaefer's theorem by showing that all potential solutions to

$$
x=\lambda A x, \quad \lambda \in(0,1)
$$

are bounded a priori, with the bound being independent of $\lambda$. With this in mind, let $x(t)$ be a solution of (3.22). Note that $x(t)$ is also a solution to

$$
\begin{gathered}
x^{\Delta \Delta}(t)=\lambda f\left(t, x^{\sigma}(t), x^{\Delta}(t)\right), \quad t \neq t_{k}, \quad k=1,2, \ldots, m, \\
x\left(t_{k}^{+}\right)=x\left(t_{k}\right)+\lambda I_{k}\left(x\left(t_{k}\right)\right), \quad x^{\Delta}\left(t_{k}^{+}\right)=x^{\Delta}\left(t_{k}\right)+\lambda J_{k}\left(x\left(t_{k}\right), x^{\Delta}\left(t_{k}\right)\right), \quad k=1,2, \ldots, m, \\
x(0)=-x(\sigma(T)), \quad x^{\Delta}(0)=-x^{\Delta}(\sigma(T)) .
\end{gathered}
$$

On one hand, we see that for $\lambda \in(0,1)$

$$
\begin{aligned}
\lambda\left\|f\left(t, x^{\sigma}(t), x^{\Delta}(t)\right)\right\| & \leq \lambda\left\{r\left[\left\langle x^{\sigma}(t), f\left(t, x^{\sigma}(t), x^{\Delta}(t)\right)\right\rangle+\left\|x^{\Delta}(t)\right\|^{2}\right]+M\right\} \\
& =\gamma\left[\left\langle x^{\sigma}(t), \lambda f\left(t, x^{\sigma}(t), x^{\Delta}(t)\right)\right\rangle+\lambda\left\|x^{\Delta}(t)\right\|^{2}\right]+\lambda M \\
& =\gamma\left[\left\langle x^{\sigma}(t), x^{\Delta \Delta}(t)\right\rangle+\lambda\left\langle x^{\Delta}(t), x^{\Delta}(t)\right\rangle\right]+\lambda M \\
& \leq r\left[\left\langle x^{\sigma}(t), x^{\Delta \Delta}(t)\right\rangle+\left\langle x^{\Delta}(t), x^{\Delta}(t)\right\rangle\right]+M \\
& =r\left\langle x(t), x^{\Delta}(t)\right\rangle^{\Delta}+M .
\end{aligned}
$$

On the other hand, by the antiperiodic boundary condition we have

$$
\int_{0}^{\sigma(T)}\left[\left\langle x(t), x^{\Delta}(t)\right\rangle^{\Delta}\right] \Delta t=\left\langle x(\sigma(T)), x^{\Delta}(\sigma(T))\right\rangle-\left\langle x(0), x^{\Delta}(0)\right\rangle=0 .
$$


It therefore follows that

$$
\int_{0}^{\sigma(T)} \lambda\left\|f\left(t, x^{\sigma}(t), x^{\Delta}(t)\right)\right\| \quad \Delta t \leq M \sigma(T)
$$

Consequently,

$$
\begin{aligned}
\|x(t)\|= & \lambda\|A x(t)\| \\
= & \| \int_{0}^{\sigma(T)} G(t, \sigma(s)) \lambda f\left(s, x^{\sigma}(s), x^{\Delta}(s)\right) \Delta s \\
& +\sum_{k=1}^{m} H\left(t, t_{k}\right) \lambda I_{k}\left(x\left(t_{k}\right)\right)+\sum_{k=1}^{m} G\left(t, t_{k}\right) \lambda J_{k}\left(x\left(t_{k}\right), x^{\Delta}\left(t_{k}\right)\right) \| \\
\leq & G_{0} M \sigma(T)+\frac{1}{2} \sum_{k=1}^{m} L_{k}+G_{0} \sum_{k=1}^{m} N_{k}+\frac{1}{2} \sum_{k=1}^{m} \delta_{i}\left\|\left(x\left(t_{i}\right)\right)\right\|+G_{0} \sum_{i=1}^{m} \zeta_{i}\left[\left\|\left(x\left(t_{i}\right)\right)\right\|+\left\|\left(x^{\Delta}\left(t_{i}\right)\right)\right\|\right] \\
\leq & G_{0} M \sigma(T)+\frac{1}{2} \sum_{k=1}^{m} L_{k}+G_{0} \sum_{k=1}^{m} N_{k}+\left[\frac{1}{2} \sum_{k=1}^{m} \delta_{i}+2 G_{0} \sum_{i=1}^{m} \zeta_{i}\right]\|x\|_{P C^{1}} .
\end{aligned}
$$

Differentiating both sides of (3.11), we can easily have

$$
\left\|x^{\Delta}(t)\right\| \leq \frac{1}{2} M \sigma(T)+\frac{1}{2} \sum_{k=1}^{m} N_{k}+\sum_{i=1}^{m} \zeta_{i}\|x\|_{P C^{1}} .
$$

Choose

$$
\begin{gathered}
N^{(1)}=\max \left\{G_{0} M \sigma(T)+\frac{1}{2} \sum_{k=1}^{m} L_{k}+G_{0} \sum_{k=1}^{m} N_{k}, \frac{1}{2} M \sigma(T)\right\} \\
N^{(2)}=\max \left\{\frac{1}{2} \sum_{k=1}^{m} \delta_{i}+2 G_{0} \sum_{i=1}^{m} \zeta_{i}, \sum_{i=1}^{m} \zeta_{i}\right\} .
\end{gathered}
$$

Thus we have by (3.18) that

$$
\|x\|_{P C^{1}} \leq \frac{N^{(1)}}{1-N^{(2)}} .
$$

Then the proof is completed. 
Example 3.4. Consider antiperiodic value problem on $\mathbb{T}=\bigcup_{k=0}^{\infty}([3 k+1,3 k+2] \bigcup\{3 k+2\})$

$$
\begin{gathered}
u^{\Delta \Delta}(t)=u(\sigma(t))+u^{\Delta}(t)^{2}+\cos t, \quad t \in[0,1], t \neq 1, \\
u\left(1^{+}\right)=u(1)+\frac{1}{8} u(1)+4, \quad u^{\Delta}\left(1^{+}\right)=u^{\Delta}(1)-\frac{1}{8} u(1)+10, \\
u(0)=-u(3), \quad u^{\Delta}(0)=-u^{\Delta}(3) .
\end{gathered}
$$

We claim (4.2) has at least one solution.

Proof. Let $T=3$ and $f(t, x, y)=x+x y^{2}+\cos t$ in Theorem 3.3. Choosing $M=4$, we have for $(t, x, y) \in[0,1] \bigcup\{2,3\} \times \mathbb{R}^{2}$ that

$$
\begin{gathered}
|f(t, x, y)|=|x|+|x| y^{2}+1 \\
\langle x, f(t, x, y)\rangle+y^{2}=x^{2}+x^{2} y^{2}+y^{2}+x \cos t \geq x^{2}+x^{2} y^{2}+y^{2}-|x|
\end{gathered}
$$

Since $\min _{v \geq 0}\left\{v^{2}-2 v\right\} \geq-1$, we have $x^{2} y^{2}+y^{2}-|x| y^{2}=y^{2}\left(x^{2}-|x|+1\right)>0$. Thus, for $\gamma=1$ and $M=2$

$$
r\left[\langle x, f(t, x, y)\rangle+y^{2}\right]+4 \geq|f(t, x, y)|, \quad \forall(t, x, y) \in[0,1] \times \mathbb{R}^{2} .
$$

Moreover, $G_{0}=9 / 4,(1 / 2) \delta_{1}+2 G_{0} \xi_{1}=(1 / 2) \times(1 / 8)+2 \times(9 / 4) \times(1 / 8)=5 / 8<1$, Then the conclusion follows from Theorem 3.3.

\section{Acknowlegments}

The research was Supported by Leading Academic Discipline Project of Shanghai Normal University (DZL805), the National Natural Science Foundation of China (10671127), the National Ministry of Education of China (20060270001), and the National Natural Science Foundation of Shanghai (08ZR1416000).

\section{References}

[1] D. Baĭnov and P. Simeonov, Impulsive Differential Equations: Periodic Solutions and Applications, vol. 66 of Pitman Monographs and Surveys in Pure and Applied Mathematics, Longman Scientific \& Technical, Harlow, UK, 1993.

[2] M. Benchohra, J. Henderson, and S. Ntouyas, Impulsive Differential Equations and Inclusions, vol. 2 of Contemporary Mathematics and Its Applications, Hindawi Publishing Corporation, New York, NY, USA, 2006.

[3] V. Lakshmikantham, D. D. Baĭnov, and P. S. Simeonov, Theory of Impulsive Differential Equations, vol. 6 of Series in Modern Applied Mathematics, World Scientific Publishing, Teaneck, NJ, USA, 1989.

[4] A. M. Samoı̆lenko and N. A. Perestyuk, Impulsive Differential Equations, vol. 14 of World Scientific Series on Nonlinear Science. Series A: Monographs and Treatises, World Scientific, River Edge, NJ, USA, 1995.

[5] S. Hilger, "Analysis on measure chains-a unified approach to continuous and discrete calculus," Results in Mathematics, vol. 18, no. 1-2, pp. 18-56, 1990.

[6] M. Bohner and A. Peterson, Dynamic Equations on Time Scales: An Introduction with Applcations, Birkhäuser, Boston, Mass, USA, 2001. 
[7] M. Bohner and A. Peterson, Eds., Advances in Dynamic Equations on Time Scales, Birkhäuser, Boston, Mass, USA, 2003.

[8] A. Cabada and D. R. Vivero, "Existence and uniqueness of solutions of higher-order antiperiodic dynamic equations," Advances in Difference Equations, no. 4, pp. 291-310, 2004.

[9] S. Aizicovici, M. McKibben, and S. Reich, "Anti-periodic solutions to nonmonotone evolution equations with discontinuous nonlinearities," Nonlinear Analysis: Theory, Methods E Applications, vol. 43, no. 2, pp. 233-251, 2001.

[10] Y. Chen, J. J. Nieto, and D. O'Regan, "Anti-periodic solutions for fully nonlinear first-order differential equations," Mathematical and Computer Modelling, vol. 46, no. 9-10, pp. 1183-1190, 2007.

[11] T. Jankowski, "Ordinary differential equations with nonlinear boundary conditions of antiperiodic type," Computers \& Mathematics with Applications, vol. 47, no. 8-9, pp. 1419-1428, 2004.

[12] K. Wang and Y. Li, "A note on existence of (anti-)periodic and heteroclinic solutions for a class of second-order odes," Nonlinear Analysis: Theory, Methods and Applications, vol. 70, no. 4, pp. 1711-1724, 2009.

[13] C. Bai, "Antiperiodic boundary value problems for second-order impulsive ordinary differential equations," Boundary Value Problems, vol. 2008, Article ID 585378, 14 pages, 2008.

[14] A. Cabada, E. Liz, and S. Lois, "Green's function and maximum principle for higher order ordinary differential equations with impulses," The Rocky Mountain Journal of Mathematics, vol. 30, no. 2, pp. 435-446, 2000.

[15] D. Franco and J. J. Nieto, "First-order impulsive ordinary differential equations with anti-periodic and nonlinear boundary conditions," Nonlinear Analysis: Theory, Methods E Applications, vol. 42, no. 2, pp. 163-173, 2000.

[16] Z. Luo, J. Shen, and J. J. Nieto, "Antiperiodic boundary value problem for first-order impulsive ordinary differential equations," Computers $\mathcal{E}$ Mathematics with Applications, vol. 49, no. 2-3, pp. 253$261,2005$.

[17] J. J. Nieto and C. C. Tisdell, "Existence and uniqueness of solutions to first-order systems of nonlinear impulsive boundary-value problems with sub-, super-linear or linear growth," Electronic Journal of Differential Equations, no. 105, pp. 1-14, 2007.

[18] Y. Xing and V. Romanovski, "On the solvability of second-order impulsive differential equations with antiperiodic boundary value conditions," Boundary Value Problems, vol. 2008, Article ID 864297, 18 pages, 2008.

[19] D. R. Smart, Fixed Point Theorems, Cambridge University Press, New York, NY, USA, 1980.

[20] Y. Xing, M. Han, and G. Zheng, "Initial value problem for first-order integro-differential equation of Volterra type on time scales," Nonlinear Analysis: Theory, Methods \& Applications, vol. 60, no. 3, pp. 429-442, 2005.

[21] J. Chen, C. C. Tisdell, and R. Yuan, "On the solvability of periodic boundary value problems with impulse," Journal of Mathematical Analysis and Applications, vol. 331, no. 2, pp. 902-912, 2007.

[22] M. Rudd and C. C. Tisdell, "On the solvability of two-point, second-order boundary value problems," Applied Mathematics Letters, vol. 20, no. 7, pp. 824-828, 2007. 